\title{
HYBRIDE IDENTITÄTEN BEI MARICA BODROŽIĆ, DANIJELA PILIC UND ANNA BAAR
}

\section{HYBRID IDENTITIES IN THE WORKS OF MARICA BODROŽIĆ, DANIJELA PILIC AND ANNA BAAR}

\author{
Ljiljana Aćimović \\ Philologische Fakultät, Universität Banja Luka; Banja Luka, Bosnien-Herzegowina \\ Filološki fakultet, Sveučilište u Banja Luci, Banja Luka, Bosna i Hercegovina
}

\section{Zusammenfassung}

In diesem Beitrag wird untersucht, wie drei $\mathrm{Au}-$ torinnen: Marica Bodrožić, Danijela Pilic und Anna Baar die Frage der Identität thematisieren. Nach der theoretischen Bestimmung der Begriffe Identität und Hybridität folgt der analytische Teil angelehnt an die von Michael Metzeltin und Thomas Wallmann zusammengestellte Auflistung der individuellen und kollektiven Identitätsmerkmale. Als Grundlage für die Analyse dienten folgende Werke: der autobiographische Prosaband Sterne erben, Sterne färben (2007) mit dem Untertitel Meine Ankunft in Wörtern von Marica Bodrožić, das autobiographische Werk Sommer vorm Balkan mit dem Untertitel Mein Leben zwischen Alpen und Adria (2015) von Danijela Pilic und der autobiographisch gefärbte Roman Die Farbe des Granatapfels (2015) von Anna Baar. In den genannten Werken wurden folgende Identitätsmerkmale behandelt: Sprache, Herkunft und Familie. In der Schlussfolgerung werden Parallelen gezogen und Gemeinsamkeiten und Unterschiede in der Konstruierung von hybriden Identitäten diskutiert.

\section{Einführung}

In diesem Beitrag wird untersucht, wie drei Autorinnen: Marica Bodrožić, Danijela Pilic und Anna Baar die Frage der Identität thematisieren. Bei allen drei Autorinnen kann man gewisse Parallelen ziehen. Alle drei stammen aus dem ehemaligen Jugoslawien: Marica
Abstract

The article analyzes how three authors: Marica Bodrožić, Danijela Pilic and Anna Baar deal with the issue of identity. The theoretical definition of the concepts of identity and hybridity is followed by an analytical part based on a list of individual and collective characteristics of the term identity compiled by Michael Metzeltin and Thomas Wallmann. The following works were used as a basis for the analysis: prose autobiographical work Sterne erben, Sterne färben (2007) with subtitle Meine Ankunft in Wörtern by Marica Bodrožić, autobiographical work Sommer vorm Balkan with subtitle Mein Leben zwischen Alpen und Adria (2015) by Danijela Pilic and novel Die Farbe des Granatapfels (2015)by Anne Baar. As characteristics of identity in the mentioned texts, the following are analyzed: language, origin and family. In the conclusion, parallels are drawn and similarities and differences in the construction of hybrid identities are pointed out.

Bodrožić wurde 1973 in Svib, in Dalmatien, geboren, emigrierte als Neunjährige nach Hessen, wo ihre Eltern schon lebten und arbeiteten. Danijela Pilic wurde 1971 in Split, auch in Dalmatien geboren, zog 1981 mit ihrem Bruder, ihrem kroatischen Vater, dem bekannten jugoslawischen Tennisspieler Nikola Pilić und ihrer 
serbischen Mutter, der ehemaligen Schauspielerin im Belgrader Atelier 212 Mija Adamović, nach München. Anna Baar wurde 1973 in Zagreb als Tochter eines österreichischen Vaters und einer dalmatinischen Mutter geboren. Die ersten vier Jahre ihres Lebens verbrachte sie mit ihren Eltern in Wien, wo sie studierten. Die Sommer verbrachte sie bei den Großeltern mütterlicherseits auf der dalmatinischen Insel Brač. 1977 siedelte die Familie nach Klagenfurt über. Die zwischenkulturellen Positionen thematisieren die Autorinnen in ihren literarischen Werken. Als Grundlage für diesen Beitrag dienten folgende Werke: der autobiographische Prosaband Sterne erben, Sterne färben (2007) mit dem Untertitel Meine Ankunft in Wörtern von Marica Bodrožić, das autobiographische Werk Sommer vorm Balkan mit dem Untertitel Mein Leben zwischen Alpen und Adria (2015) von Danijela Pilic und der autobiographisch gefärbte Roman Die Farbe des Granatapfels (2015) von Anna Baar. Aus dem vorher Gesagten kann man sehen, dass alle drei Autorinnen mindestens zwei Kulturräumen gehören: durch ihre Geburt und Herkunft sind sie mit dem ex-jugoslawischen Kulturraum verbunden, durch ihr Leben und Schreiben mit dem deutschsprachigen. Außerdem prägt die Werke von Bodrožić und Pilic die migrationsbedingte Erfahrung in fast gleichem Alter. Bei Anna Baar ist ihre multikulturelle und bilinguale Position besonders interessant.

\section{Identität und Hybridität}

Der Begriff der Identität ist eng mit der Frage „Wer bin ich?“ verbunden. Der Mensch beschäftigt(e) sich seit der Menschheit mit dieser Frage, weil er wissen wollte/will, was ihn als Individuum ausmacht und was seine Individualität kennzeichnet. Die verschiedenen wissenschaftlichen Disziplinen versuchen die Antwort auf diese Frage zu geben, aber er gibt trotzdem keine allgemein akzeptierte Definition von Identität /1/. Eines der ältesten aber zugleich einflussreichsten Identitätskonzepte entwarf der amerikanische Philosoph und Psychologe George Herbert Mead. Als sein Hauptwerk gilt der Band Mind, Self and Society from the Standpoint of a Social Behaviorist (dt. Geist, Identität und Gesellschaft aus der Sicht des Sozialbehaviorismus). Er beschäftigte sich vor allem mit der Frage, wie die menschliche Identität zustande kommt und welchen Einfluss darauf die Gesellschaft, aber auch das Denken und der Geist des einzelnen Menschen, haben.

Seine Ideen bilden wichtigste Grundlagen der Identitätsforschung, weil sie später von anderen Identitätstheoretikern aufgenommen und weitergeführt wurden /2/. Mead teilte die menschliche Identität in zwei Teilaspekte: das „impulsive Ich" (I) und das „reflektierte Ich" (me) /3/, /4/. Er war der Meinung, dass Identität sich in einem gesellschaftlichen Prozess von Strukturen und Erfahrungen konstituiert. Identität erwächst aus den Beziehungen zu anderen Individuen und unter dem Druck der Sozialisation. Da jedes Individuum zu unterschiedlichen Zeiten und in verschiedenen Rollen verschiedene Bilder von sich selbst erfährt, sie im Prozess der Sozialisation zu Selbstbildern verarbeitet und im Prozess der Interaktion als Muster für sein typisches Denken und Handeln verwendet, gibt es zahlreiche reflektierte Ichs /5/. Soziale Interaktionen der einzelnen Individuen mit anderen Menschen sind, seiner Meinung nach, sehr wichtig für die Ausbildung von sozialer Identität. Es soll betont werden, dass in seinem Identitätskonzept die Sprache, neben Spiel (play) und Wettkampf (game), eine Schlüsselrolle für die Konstituierung von Identität hat.

Nach Mead befassten sich viele Theoretiker und Kritiker mit Identitätskonzepten. Der Begriff und die mit ihm verbundenen Konzepte veränderten sich im Laufe der Zeit und hingen davon $a b$, aus welcher wissenschaftlicher Perspektive der Begriff beleuchtet wurde. Heinz Abels definiert den komplexen Identitätsbegriff folgendermaßen: „Identität ist das Bewusstsein, ein unverwechselbares Individuum mit einer eigenen Lebensgeschichte $\mathrm{zu}$ sein, in seinem Handeln eine gewisse Konsequenz zu zeigen und in der Auseinandersetzung mit Anderen eine Balance zwischen individuellen Ansprüchen und sozialen Erwartungen gefunden $\mathrm{zu}$ haben" /6/. Postmoderne Theorien der Identitätskonstruktion vertreten die „Basisannahme, dass Sinngebung und Identitätsbildung in der zersplitterten Sozialwelt zu einer privaten Angelegenheit jedes Einzelnen geworden, gewissermaßen in 'eigene Regie' übergegangen sind“ $/ 7 /$. 
Die personale (persönliche, individuelle oder subjektive) Identität steht im Mittelpunkt jeder Auseinandersetzung mit Identitätsfragen. Bei Frey und Haußer wird Identität als ein „selbsreflexiver Prozess eines Individuums verstanden“, in welchem „eine Person Identität über sich selbst herstellt, indem sie ihr Wissen, ihre Erfahrungen über sich selbst verarbeitet" und „sich selbst, ihr 'Selbst' bzw. Aspekte davon aus der Innenperspektive identifiziert" /8/. Identität ist in diesem Sinne keine Eigenschaft, die man besitzt, sondern ein veränderbarer $\mathrm{Zu}$ stand, welcher selbstreflexiv erarbeitet werden sollte /9/. Es handelt sich also um einen Prozess, welcher ständig von Änderungen und Entwicklungen bestimmt wird. In diesem Prozess geht es immer um "die Herstellung einer Passung zwischen dem subjektiven 'Innen' und dem gesellschaftlichen 'Außen'“/10/. Die äußere Dimension der "Identitätkonstruktion verweist auf das menschliche Grundbedürfnis nach Anerkennung und Zugehörigkeit" /11/. Die innere Dimension bezieht sich auf das Subjekt und ermöglicht ihm eine Selbstverortung und liefert ihm eine individuelle Selbstbestimmung /12/.

Obwohl der Identitätsbegriff eine lange geschichtliche Tradition aufweist, ist die Idee der Konstruierbarkeit von Identität relativ neu. Monika Wolting sagt, dass diese Idee „zu den elementaren Bausteinen der gesellschaftlichen Moderne“ /13/ gehört und dass "seit Mitte der Siebzigerjahre die Dynamik der Individualisierungsprozesse erheblich an Kraft zugenommen hat" /14/. Das führt dazu, dass sich in der neuesten Forschung die Auffassung von Identität ändert, und Identität ,,ist nicht als ein rein statisches oder festes Produkt zu begreifen, sondern sie stellt vielmehr ein temporäres Ergebnis der Selbst- und Fremdbestimmung eines Individuums dar" /15/.

Unterschiedliche Theoretiker führen unterschiedliche Faktoren an, die für die Konstruktion von Identität wichtig sind. Frey und Haußer reden von ,,identitätskritischen Lebenslagen" /16/ und unterscheiden: gesellschaftlich periodisierte Krisenlagen, individuelle Krisenlagen und individuelle Sinnkrisen /17/. Die erste Krisenlage ist mit "Alter" verbunden und mit der Veränderung von soziokulturellen Rollenübernahmen. Die zweite Krisenlage bezieht sich auf bestimmte Situationen in der Biographie eines Menschen, die keiner Periodisierung unterliegen, sondern unterschiedlich auftreten können. Dazu gehören zum Beispiel Unfall, Krankheit, Scheidung, Emigration. In solchen Situationen wird die persönliche Identität in Frage gestellt und eine Änderung steht an, die man akzeptieren kann oder nicht. Die dritte Gruppe, individuelle Sinnkrisen, beschäftigt sich mit Fragen des eigenen Daseins, in dem „vertraute und im Lebensraum anerkannte Muster für die Person brüchig werden, nicht mehr 'stimmten', und damit [...] selbstinduzierte Transformationsprozesse persönlicher Identität auslösen“ /18/.

Weitere wichtige Faktoren, die zur Entstehung und Konstruktion von Identität dienen, sind: Alter, Familie, Beruf, Körper und Geschlecht, Beruf, Wohnung, Herkunft oder Nationalität, die kulturelle Identität, Fähigkeiten, Gefühlszustände, Wünsche usw. /19/.

Michael Metzeltin und Thomas Wallmann listen eine Reihe von individuellen Identitätsmerkmalen auf, wie: „Namengebung, Abstammung, Alter, Geschlecht, Stellung und Funktion in der Familie, Stellung und Funktion in der Gesellschaft, Aussehen, Kleidung, physische Fähigkeiten, geistige und künstlerische Fähigkeiten, moralische Eigenschaften, Leidenschaften, Benehmen, Ideologie, Religion, Wohnung, Wohnort, Aufenthaltsort" /20/. Diese Liste erweist sich als geeignet für die Behandlung von Identitätsfragen in den hier gewählten Texten.

Die individuelle Identität setzt die Anerkennung und Zugehörigkeit voraus. Das ist mit der Existenz einer Gruppe verbunden, zu der man sich zugehörig fühlen oder von der man sich unterscheiden kann. In diesem Kontext spricht man von kollektiven Identitäten. Auch in dieser Gruppe gibt es bestimmende erkennende Merkmale, die zur Charakterisierung oder/und Identifizierung einer Gruppe oder Gemeinschaft dienen. Diese Merkmale sind: „Eigenbezeichnung, Territorialität, Herkunft, Bewusstsein einer gemeinsamen historischen Vergangenheit, Sprache, Religion und Ritten, Gesetzgebung, Verwaltung, Sitten, Konfliktaustragung und Kriegsführung, Essensgewohnheiten, Kleidung und Mode, moralische 
Eigenschaften, Leistungen in Technik, Kunst und Sport" /21/. Diese Merkmale kann man in zwei Kategorien kollektiver Identitäten gruppieren, in die kulturelle und in die nationale Identität. Diese Kategorien sind auch die Bestandteile der individuellen Identität, die ihrerseits in kollektiven Identitäten eingebettet ist. Beide entstehen in Interaktion mit der Umwelt und in Differenzierungsprozessen.

Eine bedeutende Rolle in der Analyse der hier gewählten Werke spielen die Konzepte von Transkulturalität und Transnationalität, die für die Identitätsbildung wichtig sind. Sie werden als Dimensionen von sozialen Prozessen betrachtet, in denen die Subjekte über die geographischen und nationalen Grenzen hinweg ihre Identität konstruieren. Da die Figuren der gewählten Werke mehreren kulturellen Räumen gehören und da sie sich zwischen den Kulturen positionieren, konstruieren sich ihre Identitäten als hybride Identitäten in einem Ort, den Homi Bhabha den Dritten Raum nennt.

Der aus dem Lateinischen abgeleitete und aus der Biologie entlehnte Begriff "hybrida“ bezeichnet "die Vermischung zweier oder mehrerer deutlich verschiedener Elemente, die zusammen ein Neues ergeben. Im kulturwissenschaftlichen Zusammenhang wird der Begriff insbesondere für die Vermischung von Kulturen (Hybridität) [...] verwendet" /22/. Das Konzept der Hybridität umfaßt heute „einen vielfältig auslegbaren Problemkomplex kultureller Mischformen" /23/. Neben anderen Theoretikern hat vor allem $\mathrm{H}$. Bhabha diesen Begriff unter "Anschluß an die Psychoanalyse J. Lacans und J. Derridas Begriff der différance zu einer interkulturellen Denkfigur gemacht. Diese faßt Kulturkontakte nicht mehr dualistisch, sondern entwirft einen 'dritten Raum', in dem die Konstuktion von Identität und Alterität weder als multikulturelles Nebeneinander, noch als dialektische Vermittlung, sondern als unlösbare und wechselseitige Durchdringung von Zentrum und Peripherie modelliert wird" /24/. Hybridität, „eine Mischung zwischen den divergierenden Strömungen einer Einzelkultur und eine Mischung zwischen den verschiedenen Tendenzen verschiedener Kulturen, ist also die Grundbedingung der postkolonialen Existenz" /25/. Hofmann betont, dass Bhabha den
Bereich der Kultur als ein Bereich des Transzendierens, des darüber Hinausgehenden bestimmt und behauptet: „Die Identität eines Menschen ist nicht die Herausbildung einer in ihm fest verwurzelten Anlage, sondern die produktive Auseinandersetzung mit divergierenden Tendenzen, die auf ihn einwirken. Mit Bezug auf die räumliche Dimension stellt Bhabha fest, in der Migration buchstäblich, aber in der Position ihres Sprechens auch in übertragenem Sinne besetzen die postkolonialen Subjekte einen dritten Raum, der weder der Raum einer 'Ursprungskultur' noch der zweite Raum der ehemaligen Kolonialmacht ist. Dieser dritte Raum ist der Raum des Hybriden, der an allen Räumen teil hat und doch gleichzeitig exterritorial erscheint" /26/. Das postkoloniale Subjekt ist als „ein Individuum zu begreifen, das die ihm vorliegenden Traditionslinien neu definiert und strukturiert und dabei das für es in dem individuellen Fall Bedeutsame herausstellt" /27/. Bhabha betont, dass Hybridisierung für ihn ein Prozess ist, eine Bewegung und dass in diesem Prozess sehr wichtig ist, wie die Teile in einem Subjekt miteinander und mit äußeren Kräften der Gemeinschaftsbildung in Verhandlung treten und wie diese Interaktionen stattfinden /28/. Den Dritten Raum entfaltet Bhabha als „Denkraum" über verschiedene Metaphern und Sprachfigurationen, wie zum Beispiel als „Treppenhaus“, in dem die binäre Logik verwischt, Polaritäten verschoben und hybride Identifikationen ermöglicht werden: „Das Treppenhaus als Schwellenraum zwischen den Identitätsbestimmungen wird zum Prozeß symbolischer Interaktion, zum Verbindungsgefüge, das den Unterschied zwischen Oben und Unten, Schwarz und Weiß konstruiert. Das Hin und Her des Treppenhauses, die Bewegung und der Übergang in der Zeit, die es gestattet, verhindern, daß sich Identitäten an seinem oberen und unteren Ende zu ursprüglichen Polaritäten festsetzen. Dieser zwischenräumliche Übergang zwischen festen Identifikationen eröffnet die Möglichkeit einer kulturellen Hybridität, in der es einen Platz für Differenz ohne eine übernommene oder verordnete Hierarchie

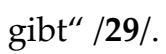

3. Konstruktion der hybriden Identitäten 
In Anlehnung an M. Metzeltins und T. Wallmanns Aufzählung werden in diesem Teil folgende Identitätserkmale behandelt: Sprache, Herkunft und Familie. Diese Auswahl wurde getroffen, um den Umfang der vorliegenden Arbeit nicht zu sprengen.

3.1 „In den Sätzen muß der Atem wohnen." Sprache als identitätsbildendes Merkmal

Durch die Aneignung von Sprache wird Identität konstruiert. Einerseits unterscheiden wir uns von anderen, indem wir eine bestimmte Sprache gebrauchen. Andererseits werden wir auf Grund des Sprachgebrauchs als eine bestimmte Sprachgemeinschaft wahrgenommen und mit dieser in Verbindung gebracht. Sprache symbolisiert auf diese Weise die Zugehörigkeit zu einer Gruppe, mit deren Hilfe eine kollektive Identität konstruiert wird.

Marica Bodrožić schreibt Gedichte, Romane, Erzählungen und Essays. Für ihr bisheriges literarisches Schaffen erhielt sie mehrere Preise und Auszeichnungen, darunter den Förderpreis für Literatur der Akademie der Künste in Berlin, den Initiativpreis der deutschen Sprache, den Literaturpreis der Europäischen Union, den Literaturpreis der Konrad-Adenauer-Stiftung und neulich den Walter-Hasenclever-Literaturpreis. Sie lebt als freie Schriftstellerin in Berlin.

In dem Erzählband Sterne erben, Sterne färben beschreibt die Ich-Erzählerin ihren Werdegang als Schriftstellerin und reflektiert an vielen Stellen über die Sprache(n). In diesem Buch dominiert der monoperspektivische Erwachsenenblick und die Narration verläuft nicht chronologisch. Schon am Anfang des Buches verweist das reflektierende Ich auf seine spezifische Position. Einerseis bezieht sich dieser Verweis auf seine slawische Herkunft, andererseits auf seine Verbundenheit der deutschen Sprache gegenüber: „Die Selbstverständlichkeit, mit der die Wälder des Slawischen in mir liegen, wird mit erst im Schreibengehen bewußt. Dieses Unterpfand, das immer aus der ersten Sprache herauftönt und mich endlich zu jemand macht, der etwas von sich sagen kann. Aber erst in der deutschen Sprache wird mein eigenes Zuhause für mich selbst hörbar" /30/. Die biographische
Bedeutung der Auswanderung wird hier durch die Fremdheitsposition reflektiert. Die Fremdheitserfahrung konstituiert sich zunächst als eine Konkurenzbeziehung zweier Welten an den Grenzen der Sprache und Sprachlosigkeit als Ausdruck innerer Konflikte und Zerrissenheit. Die Verbundenheit der deutschen Sprache gegenüber könnte man als eine eindeutige Verortung der Identität lesen. Aber im weiteren Verlauf des Textes zeigt sich, dass die Ich-Erzählerin durch Reflexionen und Erinnerungen versucht die Frage ihrer eigenen Identität zu beantworten. Ihre Muttersprache bezeichnet sie sehr selten als Muttersprache, umso mehr als ihre „erste Sprache“, oder als „die Sprache ihrer Kindheit". Andererseits gibt sie dem Deutschen den Vorrang: "Aber irgendwann wurde die deutsche Sprache ein Terrain des Wissens, des Fragens auch, und damit kehrte etwas wie Entschiedenheit in mein Leben ein. Nur im Deutschen ließ es sich präzise träumen. Das Fließen der Sprache wurde zur Gewißheit, zur Mathematik des sich aufbahrenden Geheimnisses, so, als wolle das Unerlöste, das von den Wunden der Kindheit umzäunte Gebiet, hinausgelangen, hinaus aus sich, aus mir, als seinem Statthalter, hinein in die Welt, in der die Namen und Wörter atmen dürfen, ohne eine Begründung dafür haben zu müssen, ohne Rechtfertigung und auch ohne eine Absicht" /31/.

Die Kindheit ist aus ihrer Sicht sehr wichtig für die Identitätskonstruktion: „Heute denke ich oft, daß die Gesetze des eigenen Lebens bereits gebündelt in der Kindheit schon gewirkt haben, als Kern der eigenen Biographie, die wir in den Sterntaschen unserer Selbst mitgebracht haben. Ist sie nur weit und tief genug begangen worden, wächst sie zu einem eigenständigen Sterngebiet heran. Jede Kindheit trägt das Erbe der Sterne in sich, strahlt sie aus uns heraus, hinein in die Wörter und Sätze, Gesichter, Brustkörbe und Lieder der Menschen. Die einstigen Jugoslawen, ob sie es wollten oder nicht, haben ein ganzes Sternengepäck aus dem Kosmos mitgebracht"/32/.

Dieses „Erbe der Sterne der einstigen Jugoslawen“ spielt die entscheidende Rolle in drei Begegnungen mit den "Fremden“. Als in Rom eine junge Frau die Erzählerin auf Englisch angesprochen hat, erkannte sie aufgrund 
"dieses Erbes der Sternen“, „daß sie eine von uns ist, eine von uns, dachte ich damals noch immer, obwohl es schon 1998 war und ich offiziell nicht das Recht gehabt habe, so zu denken" /33/. Es stellte sich heraus, dass die junge Frau eine serbische Studentin aus Belgrad sei, die in Rom Kunstgeschichte studierte und Sehnsucht nach Belgrad und nach der anderen Seite der Adria hatte. Obwohl die Erzählerin behauptet, dass erst in der deutschen Sprache ein eigenes Zuhause für sie hörbar wird /34/, zeigt sich in dieser Begegnung das Erbe der ersten Sprachidentität, denn sie erkannte in dem Englischen der jungen Studentin ihren "eigenen Jugo-Ton und sie gleich in unserer Sprache anredete, ohne ihre Antwort auf meine Frage, woher sie sei, abzuwarten" /35/. Diese Begegnung hinterließ unerwartet einen tiefen Eindruck auf sie: "Ihre Worte klangen in mir nach wie der Widerhall eines tiefen Gebetes"/36/.

Ähnlich fühlte sie sich in Paris, als sie Plakate bemerkt hatte, die ein Konzert von Goran Bregović ankündigten. Dazu notiert sie folgendes: „Es fühlte sich an, als würden hier, an einem mir noch weitgehend fremden Ort, an dem ich eine neue Sprache erlernen wollte, meine ersten Wörter, meine Herkunft auf mich einreden, auf die Art der Stummen sprach all das zu mir, nahm mich bei der Hand, und an den darauffolgenden Tagen sah ich die Plakate überall“ /37/. Die dritte Begegnung ist die Begegnung mit der alten Frau am Frankfurter Hauptbahnhof, die, mit ihrem nach hinten gebundenen blumenbestickten Kopftuch, am Perron stand und die sie mit den einfachsten Worten segnete /38/. An allen diesen Stellen sieht man ihre Hybridität.

Die Bedeutung der ersten Sprache zeigte sich bei der Erzählerin auch, als sie aus der zweiten in eine dritte Sprache zog: „Erst das Loslassen zeigte mir, was ist bereits besitze. Es kommt mir aus der Rückschau so vor, daß die wartende dritte Sprache, das wartende dritte Land mir Brücke gewesen ist für den sich schließenden Kreis, für das schwierige Anerkennen meiner Herkunft in mir selbst" /39/.

Die Verbundenheit mit der ersten Sprachidentität und mit dem Land der Kindheit tritt in den Vordergrund beim Erlernen des Französischen: „Als ich begann, das Französische zu erlernen, bemerkte ich, wie unter der feinen deutschen Wetterwörterschicht, unbekümmert und fern jeder Formhaftigkeit, mein unmitellbarer dalmatinischer Dialekt lebte, einzelne herzegovinische Wörter in meinen Gedanken auftauchten, das Kroatische, das einstige Serbokroatische, auch Osmanisch klingendes aus der Gegend meiner Mutter, die in der Nähe von Mostar zur Welt gekommen ist. Was in mir als Kind gefiebert und gearbeitet hatte, zeigte sich hier" /40/. Daraus ergibt sich als völlige Überraschung die Feststellung: „Nicht das Deutsche lag also als Sprachgerüst für die neue Sprache in mir, sondern $\mathrm{zu}$ meiner großen Überraschung am Ende doch die Muttersprache. Die erstsprachlichen Wörter redeten förmlich auf mich ein, traten hier hervor, frohlockten dort. Diese Entdeckung war ein Glückserlebnis für mich. Ein schockhaftes" /41/ und endlich die Erkenntnis ihrer hybriden sprachlichen Identität: „Das Durchschreiten beider Sprachen kam mir manchmal vor wie zweifaches Leben, wie zwei autonom nebeneinander wirkende Lebensspuren, die $\mathrm{zu}$ verbinden mir nur im Schreiben gelang. Im gelebten Alltag verweigerte sich diese Verschmelzung, als gehöre zu jeder Sprache ein ganz eigenes, eigenständig arbeitendes Herz, das alleinsprechend ist, und als müsse alles einzeln gelebt werden, bis die Einheit begönne. Wer oder was diesen Zeitpunkt bestimme, bleibe bis auf weiteres ein Geheimnis" /42/.

Es stellt sich heraus, dass nicht nur ihre gegenwärtige Identität hybrid ist, sondern auch ihre erste Sprache. Sie war „immer schon etwas Hybrides, etwas durch und durch Unvollkommenes, aus Kreuzungen und Ahnungen bestehendes Gemisch aus dem dalmatinischen Dialekt, der Sehnsucht nach einem hochkroatischen Sprachfluidum, wie es die Leute in der Hauptstadt um sich herum verbreiteten, aus herzegovinischen Wortendungen, Redensarten von hier, Redensarten von dort, eine Art, mit den Wörtern zu lachen, eine andere, mit ihnen zu schweigen, verschwiegen zu bleiben, wie es die Gebirgsgegend nahelegt; zudem hieß das Ganze Serbokroatisch, hielt größere Räume offen, verschiedene Wörter für Zug gab es, und wenn es das Glück gab, dann weil es viele Wörter für eine Sache gab" /43/.

Ähnliche Sätze über die Zerrissenheit des Ich in der Sprache kann man in Anna Baars 
2015 im Wallstein Verlag erschienenem Erstlingroman Die Farbe des Granatapfels lesen. Der Roman stand drei Monate auf Platz 1 der ORFBestenliste. Mit einem Auszug aus dem Manuskript nahm die Autorin am Ingeborg-Bachmann-Preis teil. Für den zweiten Roman Als ob wir träumend gingen erhielt sie den TheodorKörner-Preis und neulich den Humbert-FinkPreis. Anna Baar schreibt Erzählungen, Kurzgeschichten und Romane. Sie lebt als freie Schriftstellerin in Klagenfurt und Wien.

Im Roman Die Farbe des Granatapfels erzählt die Ich-Erzählerin, die stark autobiographisch geprägt ist, über ihre Kindheit und Jugend und über das Heranwachsen zwischen zwei widesprüchlichen Kulturen und Denkarten. Einserseits steht die arhaische Welt eines Fischerdorfs im dalmatinischen Mutterland, andererseits das Leben in einer österreichischen Stadt im Vaterland. Dieses Dazwischenstehen, oder der Bhabhas Therminologie nach das Leben im Dritten Raum, ist das Thema dieses Romans.

Im ganzen Buch unterscheidet die Erzählerin zwischen der Muttersprache (darunter versteht sie eigentlich die Sprache der Mutter) und der Vatersprache (die Sprache des Vaters). Es kommt dazu noch die dalmatinische Mundart ihrer Großmutter Nada. Da sie jahrelang die Sommermonate bei ihrer Großmutter mütterlicherseits verbringt, ist sie der beiden Sprachen mächtig. Aber in der Kindheit ging es nicht so leicht. Das Kind, wie die erwachsene Erzählerin sich selbst in den Erinnerungen an die Kindheit bezeichnet, war oft sprachlos. Vielleicht ist das eine Folge der inneren Zerrissenheit des jungen Wesens zwischen zwei Welten: „So blieb es reglos, um wieder ins Nichts oder - wer wusste das schon - in eine andere Welt zu starren, schien nicht zu bemerken, wenn man es anrief, und niemand fand je heraus, was sich in seinem Inneren zutrug, denn seine Antwort auf die Frage, woran es gerade denke, war immer die gleiche: Nichts - und manchmal nur den Mund zum Strich gepresst und nur ein Schulterzucken, als hätte es ihm die Sprachen verschlagen, die Muttersprache, die Vatersprache, die Engelszunge auch"/44/. Die Zerrissenheit des Kindes ist um so größer, da die Großmutter das andere Land, das Vaterland, die Heimat der Enkelin, verächtlich „Esterraich“ /45/ nennt, wo "Ibermenschen" /46/ leben und wollte aus ihm "ihr Kind" /47/ machen. Mit der Zeit entwickelte das Kind eine heimliche Strategie auch während der Sommermonate in Verbindung mit der Vatersprache, die es vermisste, zu sein: es saß auf der Gartenmauer und lauschte den Gesprächen der Fremden: „Die Fremden freilich bemerkten nicht, dass man horcht und lauert, sich an jedem Wort erbaut, an den Tonfolgen und Melodien der Fremdensprache, der Vatersprache, der Mördersprache, nach der einem das Maul gewachsen ist, nach der sich die Zunge wälzt und die Lippen schürzen, aber die man sich verbeißen muss, um nicht mitgemeint zu sein mit den Fremden, mit den Schlechten, denn man ist von Nadas Schlag, von ihrem Stern" /48/ oder es schrieb, denn im Schreiben konnte man die Vatersprache gebrauchen, die leibliche Sprache, in der man für Wochen schwieg und doch fortwährend dachte, träumte und empfand $/ 49 /$. Aus diesen Sätzen geht hervor, dass das Kind nur eine sprachliche Identität konstruiert, die durch die Vatersprache. Erst Jahre später, als die Kindheit hinter der Erzählerin ist, wird sie mit Wehmut schließen, dass dieses Hin und Her zwischen den Sprachen tiefe Spuren hinterlassen hat und dass ihre sprachliche Identität eigentlich hybrid ist: „Ich wünschte mich zurück in die Obhut der Vatersprache, die mir leichter von den Lippen ging als Nadas Mundart, in der ich wohl fühlte und sang und fluchte, der mein Gemüt aber nicht gewachsen war und die mich mit meinem scharfen, kehligen $R$ schnell als Fremde entlarvte. Der Wechsel zwischen den Sprachen war mehr als der Wechsel des Zungenschlags, mehr als ein anderes Lippenschürzen. Er war eine rätselhafte Metamorphose, ein neues Lied, das man anstimmte, um den Gesetzen eines anderen Denkens und Fühlens zu folgen /50/. In diesem Satz sieht man am besten, dass die Funktion der Sprache viel mehr als bloße Vermittlung von kognitiven Inhalten ist. An Sprache sind gewisse Emotionen, Erlebnisse, Erinnerungen gebunden, die die Identität eines Individuums prägen. Dessen wird die Erzählerin beim Ausbruch des Krieges in Jugoslawien bewusst, wann viele Flüchtlinge „von dort unten“ 
/51/ in ihre österreichische Stadt ankommen. Sie mischt sich mit ihnen „beseelt vom Klang ihrer Sprache, die mich vertraut umspann mit ihren Worten, Modulationen, Silben, die, wo sie die eine Sehnsucht stillten, sofort eine andere, tiefere aufrissen, und wie ich so horchte, verdichteten sich alle Flüche und Klagen zu einem Zirpen, zum fröhlichen Gezwitscher, das an den Hochsommerabenden aus den Schwalbennestern in der Kapelle des heiligen Antonius drang, [...] und endlich die Gewissheit, dass mir die Seele da beheimatet war, in der Muttersprache, die ich immer noch sprach wie ein Kind und die ich mir im Vaterland versagte, um nicht gehört zu werden, um nicht verhöhnt $\mathrm{zu}$ werden, um nicht mitgemeint zu sein mit denen von dort unten, nicht mitgemeint zu sein in den Schimpfworten, den Worten für das Böse, den Worten für das Fremde in mir, das nur Nada liebte" /52/. In diesen Worten sieht man am besten ihr Dazwischenstehen, ihr Leben zwischen zwei Kulturen und zwischen zwei antagonistischen Welten - ihre Hybridität.

3.2 ,Deine Herkunft kann dir niemand nehmen“. Herkunft als identitätsstiftendes Merkmal

Die Frage der Herkunft stellt eine der wichtigsten Identitätsfragen dar. Diese Frage ist eng mit der Frage der Heimat verbunden. In ihrer Autobiographie schreibt Danijela Pilic an vielen Stellen über die Herkunft und nennt es Wurzeln.

Danijela Pilic erzählt in ihrer 2015 veröffentlichen Autobiographie von ihrem Leben, von dem Leben ihrer Familie, von dem Land, in dem sie geboren wurde und welches es nicht mehr gab, Jugoslawien, von ihrer Heimat und von dem Leben im Exil, das irgendwann das Zuhause geworden ist. Sie lebt und arbeitet in München.

Deutschland nennt sie „mein neues Land“ /53/ und Jugoslawien "meine Heimat" /54/ oder "mein alter Staat" /55/ und schon am Anfang berichtet sie, wie sie als Achtzehnjährige die Entscheidung für die deutsche oder für die jugoslawische Staatsbürgerschaft treffen musste. Ihre Weigerung, den deutschen Pass anzunehmen, erklärt sie damit, dass sie eine Jugoslawin ist und dass sie aus Jugoslawien stammt, worauf sie die Antwort von ihren Eltern bekommt, dass ihr niemand ihre Herkunft nehmen kann /56/. Sie nahm die deutsche Staatsangehörigkeit an, aber mit einem Schuldgefühl, ihre Heimat im Stich gelassen zu haben. Diese Situation diente als Ausgangspunkt für die Erkenntnis über die Wichtigkeit der Wurzeln: „Sie bestimmen nicht nur, woher du kommst, wie du aussiehst, woran du dich erinnerst, wie rastlos du bist, wie du alterst, sondern auch, was du bist, warum du bist, wo du bist, und außerdem: wohin du gehst, wie häufig du das Ziel änderst und wie leicht dir Letzteres fällt. [...] Man ist mehr als die Summe seiner Teile, doch was ist, wenn sich die Teile völlig und konstant verändern" $/ 57 /$.

Obwohl die Erzählerin im ganzen Buch darauf besteht, dass Jugoslawien ihre Heimat ist, das Land ist, aus dem sie stammt, wird ihr nach Jahren in Deutschland klar, dass Split nicht mehr ihr Zuhause war, weil sich ihr Leben woanders abspielte, und dass man verschiedene Zuhause aufeinanderpacken kann und dass „die Fahrt nach München zur Fahrt nach Hause [wurde], und es war nichts Dramatisches daran, nichts Einschneidendes und nichts Trauriges. Es war einfach so. Ich hatte nichts verloren, nur Sprachen und Koordinaten gewonnen. Ich hatte ein neues Zuhause, wer weiß für wie lange, doch diese meine arme kleine Heimaterde würde immer da sein für mich, so wie sie war, die schönste für mich" /58/.

Aus dieser Erkenntnis geht die Schlussfolgerung hervor, dass ihre Identität nicht mehr singulär ist und dass sie in sich selbst auch einen deutschen Teil hat /59/ und dass ihr Herz halb in einem halb in einem anderen Land ist /60/. Daraus ergibt sich ihre Hybridität, die sie folgendermaßen formuliert: „Wie soll ich mich bezeichnen? Tausende von Konversationen darüber haben dies nicht eindeutig klären können, ich weiß es immer noch nicht: als Südslawin, Deutsche, Münchnerin, Ex-Berlinerin, Europäerin? Oder Ex-Jugoslawin? Oder, weil es viele so gern genau wissen wollen, halbe Serbin, halbe Kroatin, seit ihrem zehnten Lebensjahr in Deutschland lebend, seit dem achtzehnten eingebürgert, die ić-Endung in ihrem Namen (auch wenn sie schließlich zum -ic eingedeutscht wurde) immer mit sich tragend?" /61/. 
Ähnlich wie die Ich-Erzählerin in Anna Baars Roman die Zerrissenheit in Bezug auf die Sprache erlebt, erlebt sie auch die Zerrissenheit in Bezug auf die Herkunft. Sie unterscheidet, besonders in Kinderjahren, zwischen dem Vaterland (worunter sie das Land des Vaters) versteht und dem Mutterland (das Land der Mutter). Außerdem machen auch weitere Bezeichnungen: das andere Land, das Fremland, das Drübenland, das Land der Eltern, die innere Zerrissenheit des Kindes immer tiefer, und es fragt sich sehr oft, wo es hingehört: „In manchen Momenten hatte es ein Bewusstsein, Ich bin, bin tatsächlich!, und im nächsten Augenblick ein Verdacht, gleichsam fehl am Platz zu sein, auch im anderen Sein beheimatet [...] Vielleicht auch fehl am Platz, weil es in dieser anderen Sprache dachte und träumte, auch wenn es ganz im Hier war" /62/.

Seine Großmutter Nada versuchte immer, dem Kind das ihrige zu übertragen, ihre Betrachtungen und Überzeugungen und verstand nie, dass ",das andere Land nicht nur ein Anderswo, sondern sein anderes Zuhause war" /63/. Das Kind sehnt sich nach dem Vater und nach der Mutter und vielleicht war die "Sehnsucht nach dem anderen Land nur ein $\mathrm{Zu}$ fluchtswunsch“ /64/, ein Wunsch ",endlich aus dem Zwischenweltenwandler ein Anwesender [zu werden], vielleicht ein Ankommen, da wie dort" /65/. Und obwohl es immer versuchte, zu verstehen, wo es hingehört und auch zwischen diesen zwei Welten zu balancieren, schien es „unmöglich, beiden Welten angehörig zu werden, aber man konnte tun, als ob, und das Lachen gelang mühelos [...] Da wie dort lautete der Auftrag, nur ja nicht nach den anderen zu geraten, obwohl man da wie dort stets anders blieb" /66/, woraus die in der Zwischenzeit erwachsene Erzählerin die Schlussfolgerung zieht, dass sie keiner von den beiden Welten angehört und dass sie in beiden Welten als fremd empfunden war: „Das Fremdsein blieb unvermeidlich, hier wie dort - unter jenen, die mir das Vaterland absprachen und den Winter verleideten, wie unter jenen, die mir die Muttersprache verübelten, die Banditensprache, die denen eine Mördersprache war" /67/ und dass sie das bleiben will, was sie immer war: „ein Zaungast. Ein anderer Platz steht den Fremden nicht $\mathrm{zu}^{\prime \prime} / 68 /$.

In diesem Kontext lässt sich die Grenze, oder besser gesagt der Tunnel zwischen zwei Ländern, sehr gut als Bhabhas Dazwischenraum, als Dritter Raum, betrachten: „Das Hinundher ist mir zur Gewohnheit geworden, das Zwischenreich der scharf bewachten Grenzen, der hohe Pass. Wer von da nach dort wollte, musste durch die Geisterbahn tief im Inneren des Gebirgkamms [...] Fuhr man vom Vaterland aus in den Tunnel ein, war die Röhre gut beleuchtet. Das Mutterland begann auf halbem Wege, da, wo man in die Düsternis rußiger Wände und diesiger Beleuchtungskörper tauchte. Der Punkt hatte etwas Magisches. Es schien, als müsse man ihn minutiös vorherberechnen, ihn feststellen, ihn sich einprägen Jetzt!, - diesen einen Schritt von einem Erdteil zum anderen, der immer auch eine Verrückung, ein Übertritt in eine andere Wirklichkeit, in ein anderes Leben" /69/. Aus diesen Beispielen geht deutlich hervor, wie schmerzhaft die Erzählerin ihre Kindheit und ihr Leben wahrnimmt und auf welche Weise sie ihre zwischenkulturelle Identität konstruiert.

3.3 „Was bliebe ohne sie von unserer Welt?“ Familie als identitätsbildendes Merkmal In allen drei Texten spielen Familie und familiäre Beziehungen eine wichtige Rolle für die Konstruktion von Identität. Das gilt besonders für die Beziehung zwischen Großeltern und Enkelkindern. Pilic verweist in ihrer Autobiographie auf die Rolle ihrer Großeltern mütterlicherseits baka (Oma) Mara, die für sie der sicherste Hafen ihrer Kindheit war /70/ und deda (Opa) Petar, der ihr die Farben beibrachte /71/. Bei ihnen verbrachte sie, während ihre Eltern auf Reisen waren, die wunderbarste Zeit ihrer Kindheit. Auch auf die kroatischen Großeltern none (Oma) Danica und dida (Opa) Krsto erinnert sie sich gerne. Mit der none Danica verbindet sie auch der Name, was für die individuelle Identität sehr wichtig ist, weil ihr Name der Ausgangspunkt für den Namen der Autorin war /72/. Schon diese unterschiedlichen Benennungen lassen erkennen, dass sie in einer multiethnischen Familie aufwuchs. Aber auf die 
Tatsache, dass sie aus einer "Mischehe“ stammt, kommt sie eigentlich erst in Deutschland, als sie ein Formular bekommt und ankreuzen sollte, was man war. Sie wusste nicht, was sie ankreuzen sollte: „Darüber hatte ich mir noch nie Gedanken gemacht. Natürlich wusste ich, dass meine Mutter aus Novi Sad stammte und in Belgrad gelebt hatte und Serbin war, aus der Vojvodina, ebenso war mir bekannt, dass mein Vater aus Split kam und Kroate war, aus Dalmatien. Doch ich wusste nicht, nicht aktiv, dass sie Serbin ist, und auch nicht, dass mein Vater Kroate war. Ich hätte es gewusst, hätte ich jemals darüber nachgedacht, doch das habe ich nicht. Es wurde nie ausgesprochen, nie festgehalten, weil es nicht wichtig war. Ich kam aus Jugoslawien. Aber war ich auch Jugoslawin?" /73/ Da sie sich nicht entscheiden konnte, hat sie das Formular nie abgegeben. Aber, es blieb die Erkenntnis, dass sie eine Jugoslawin war, und auf diese Erkenntnis kam sie erst 1981 in München /74/ Diese Erkenntnis wird sich später als sehr wichtig bei der Bestimmung ihrer Identität zeigen.

Bei Bodrožić hat auch die Familie eine wichtige Rolle für die persönliche Identitätsbildung. Auch hier entpuppt sich die GroßvaterEnkelin-Beziehung sehr wichtig für die Konsturktion von Identität. Schon am Anfang des Buches wird die Rolle des Großvaters betont. Er dient als Ersatzfigur für die abwesenden Eltern, für das kroatische Zuhause der Kindheit und figuriert als wichtigste Person im Leben der Erzählerin vor ihrer Auswanderung nach Deutschland. Diese Großvater-Enkelin-Beziehung ist wichtig, da sie nicht nur mit Liebe und Sehnsucht zu tun hat, sondern sie dient auch als Motivation für die erzählende Identitätskonstruktion: „Mein erstmalig bewußt erlebender Verwandter war nicht ein Mensch. Es war das Gesicht meines Großvaters. Dieses Bild der unsterblichen Wangen und der in meiner Herzerinnerung fortlebenden blauen Augen habe ich nie in meiner ersten Sprache erinnert. Im Deutschen meldete es sich gleich einem Mitbewohner meines Hauses an und kehrte so lange beharrlich zu mir zurück, bis ich zu einem Stift griff und es zu beschreiben versuchte. Es ist so lange geblieben, bis alles erzählt zu sein schien, was die Farben von Wangen und Augen mir gesagt hatten, und bis ich verstand, daß der Tod dafür zuständig ist, uns an das gelebte Leben zu erinnern. Er erinnert auch an das Versäumte, an das uns vom Leben Trennende, die Trägheit auch, die uns von der eigentlichen Fähigkeit zu empfinden abhält. Zu empfinden: in der Sprache selbst zu lieben" /75/.

Die Großvaterfigur hat zusätzlich eine spezifische Rolle. Diese Figur fungiert als eine Verbindung zu den Verstorbenen, besonders zur frühverstorbenen Großmutter Mara, deren Name, wie bei Pilic, auch der Ausgangspunkt für den Namen der Erzählerin ist, und auf diese Weise wichtig für die Konstruktion der individuellen Identität.

Ganz anders konstruiert Anna Baar die Beziehung der Ich-Erzählerin zu ihrer Großmutter Nada. Die Großmutter-Enkelin-Beziehung ist stark dadurch gekennzeichnet, dass Nadas Schwiegersohn und der Vater der Enkelin dem Volk von „,drüben“ angehört. Diese von „drüben“ waren die Feinde ihres Volkes und hatten im Krieg ihre Schwester ermordet, was sie weder vergessen noch verzeihen konnte. Ihr Hass ist so groß, dass sie sogar die Herkunft ihres Schwiegersohnes verfälscht. Sie brachte dem Kind ihre Vorstellungen und Denkarten bei, mit dem Ziel, aus ihm das ihrige Kind zu machen. Sie nannte es ihr ganzes Glück, ihr Einundalles, ihr Kind /76/. Die erwachsene IchErzählerin bezeichnet ihre Beziehung retrospektiv als Trauerspiel, an dem sie sich, seit sie sich erinnern kann, schadenklug und kleinlaut entlangtastet und in ihre Fallstricke glitscht /77/. Die Erinnerungen kreisen um viele Ereignisse aus der Kindheit und Jugend und beleuchten die komplizierte Beziehung zwischen der Großmutter und der Enkeltochter.

Und obwohl Nada mit der Zeit ihren Eifer vervielfachte, das Kind für das ihrige zu erwärmen, musste sie traurig feststellen, dass ihre geliebte Enkelin immer mehr „Wesensmerkmale zeigte, die sie der anderen Seite zuschrieb"/78/. Trotzdem liebte sie das Kind und die Enkelin liebte trotz allem die Großmutter.

Und jetzt im Moment, als sich Nadas Leben dem Ende nähert, fragt sich die Enkelin, ob ihre Großmutter ihre Angst errät, sie entbehren zu müssen und „was bliebe ohne sie von unserer Welt?" /79/ und gibt als Antwort „Nichts bliebe mir ohne sie [...] Ein Flecken Erde nur, seelenlos mit einem Mal, unberührt von 
unserem Geschick, gleichgültig auch gegen das Kind, das da heranwuchs, an Nadas Brüste und Brandstätten kroch, sich da wieder und wieder verbrannte, das gebrannte Kind, das das Feuer nicht scheute, nicht weil es aus dem Schaden nicht klug geworden wäre, sondern weil es sich der Gefahr aussetzen musste, um sich in Nadas Fühlung zu wissen, sich in Sicherheit zu wissen vor jenen viel größeren Gefahren, vor denen sie dauernd warnte" /80/. Diese komplizierte Beziehung erweist sich als eine wichtige Komponente der Identitätsfigur der Enkelin.

\section{Schlussfolgerung}

In den hier analysierten Werken kann man viele Parallelen ziehen und viele Gemeinsamkeiten feststellen. Alle Texte sind autobiographisch geprägt und werden von einer Ich-Erzählerin präsentiert. In allen Narrativen haben Erinnerungen eine wichtige Rolle. Eben durch die Erinnerungen wird die Identität konstruiert. Die Ich-Erzählerinnen in den Texten von Bodrožić und Pilic verbindet die Erfahrung der Migration. In diesem Sinne unterscheiden sie sich von Anna Baars Erzählerin, die multikulturell und zweisprachig aufwächst. Als identitätsbildende Merkmale werden Sprache, Herkunft und Familie behandelt. Im Bezug auf die Sprache lässt sich schließen, dass alle Erzählerinnen ihre Mehrsprachigkeit als ein Vorteil betrachten. Aber den Prozess der Aneignung der Mehrsprachigkeit erleben sie mehr oder weniger schmerzhaft und eben in diesem Prozess konstruieren sie Bhabhas Dritten Raum, einen Dazwischenraum, in dem die Sprachen zusammentreffen und aus ihnen hybride Identitäten machen. Was die Herkunft angeht, zeigt sich Pilics multiethnische Herkunft besonders interessant im Ausland und zwar in Verbindung mit dem Ausbruch der Kriege auf dem Balkan. Von diesen Kriegen wird auch bei Bodrožić und Baar erzählt. Ihre multikulturelle und hybride Herkunft thematisiert äußers schmerzhaft Anna Baars Erzählerin. Auch im Bezug auf die Familie als identitätsbildendes Merkmal findet man Parallelen in den Großeltern-Enkelkindern-Beziehungen. Alle Texte kennzeichnen außerdem viele serbokroatische Worte oder Sätze, oft ohne deutsche Übersetzung, was die Hybridität dieser Texte zusätzlich markiert.
Notes

/1/ Frey, H. P., Haußer, K. (1987). Entwicklungslinien sozialwissenschaftlicher Identitätsforschung. In: Frey, H. P., Haußer K. (Hg.): Identität. Entwicklungen psychologischer und soziologischer Forschung. Stuttgart: Enke. (Der Mensch als soziales und personales Wesen. Bd. 7), S. 3.

/2/ Lohauß, P. (1995). Moderne Identität und Gesellschaft. Theorien und Konzepte. Opladen: Leske + Budrich, S. 9.

/3/ Lohauß, P. (1995). Op. cit., S. 15.

/4/ Abels, H. (22017). Identität. Über die Entstehung des Gedankens, dass der Mensch ein Individuum ist, den nicht leicht zu verwirklichenden Anspruch auf Individualität und Kompetenzen, Identität in einer riskanten Moderne zu finden und zu wahren. 3., aktualisierte und erweiterte Auflage. Wiesbaden: Springer VS, S. $212 \mathrm{ff}$.

/5/ Abels, H. (32017). Op. cit., S. 213.

/6/ Abels, H. (32017). Op. cit., S. 200.

17/ Eickelpasch, R., Rademacher, C. (2004). Identität. Bielefeld: transcript Verlag, S. 11.

/8/ Frey, H. P., Haußer, K. (1987). Op. cit., S. 4.

/9/ Frey, H. P., Haußer, K. (1987). Op. cit., S. 11.

/10/ Keupp, H. (o. J.). Identität. Verfügbar unter: https://www.spektrum.de/lexikon/psychologie/identitaet/6968 [Zugriff am: 15. 4. 2020].

/11/ Keupp, H. (o. J.). Op. cit.

/12/ Keupp, H. (o. J.). Op. cit.

/13/ Wolting, M. (2017). „Identität kann nur als ein Problem existieren" - Zu den Identitätskonstruktionen in der Gegenwartsliteratur. Einleitung“. In: Wolting, M. (Hg.): Identitätskonstuktionen in der deutschen Gegenwartsliteratur. Göttingen: V\&R unipress, S. 9.

/14/ Wolting, M. (2017). Op. cit., S. 9.

/15/ Wolting, M. (2017). Op. cit., S. 10.

/16/ Frey, H. P., Haußer, K. (1987). Op. cit., S. 12.

/17/ Frey, H. P., Haußer, K. (1987). Op. cit., S. 12.

/18/ Frey, H. P., Haußer, K. (1987). Op. cit., S. 13.

/19/ Frey, H. P., Haußer, K. (1987). Op. cit., S. $14 \mathrm{ff}$.

/20/ Metzeltin, M., Wallmann, T. (2010). Wege zur Europäischen Identität. Individuelle, nationalstaatliche und supranationale Identitätskonstrukte. Berlin: Frank \& Timme Verlag für wissenschaftliche Literatur, S. 13-14.

/21/ Metzeltin M., Wallmann, T. (2010). Op. cit., S. 49.

/22/ Nünning, A. (Hrsg.). (2005). Grundbegriffe der Kulturtheorie und Kulturwissenschaften. StuttgartWeimar: Verlag J. B. Metzler, S. 67.

/23/ Nünning, A. (Hrsg.). (2005). Op. cit., S. 68.

/24/ Nünning, A. (Hrsg.). (2005). Op. cit., S. 68.

/25/ Hofmann, M. (2006). Interkulturelle Literaturwissenschaft. Eine Einführung. Paderborn: Wilhelm Fink Verlag, S. 28. 
/26/ Hofmann, M. (2006). Op. cit., S. 29.

/27/ Hofmann, M. (2006). Op. cit., S. 30.

/28/ Bhabha, H. K. (2012). Über kulturelle Hybridität. Tradition und Übersetzung. Aus dem Englischen von Kathrina Menke. Wien-Berlin: Verlag Turia+Kant, S. 66.

/29/ Bhabha, H. K. (2011). Die Verortung der Kultur. Mit einem Vorwort von E. Bronfen. Übers. von M. Schiffmann und J. Freundl. Tübingen: Stauffenburg Verlag, S. 5.

/30/ Bodrožić, M. (2016). Sterne erben, Sterne färben. Meine Ankunft in Wörtern. München: btb Verlag, eBook, S. 8.

/31/ Bodrožić, M. (2016). Op. cit., S. 14.

/32/ Bodrožić, M. (2016). Op. cit., S. 32-33.

/33/ Bodrožić, M. (2016). Op. cit., S. 33.

/34/ Bodrožić, M. (2016). Op. cit., S. 8.

/35/ Bodrožić, M. (2016). Op. cit., S. 34.

/36/ Bodrožić, M. (2016). Op. cit., S. 34.

/37/ Bodrožić, M. (2016). Op. cit., S. 40.

/38/ Bodrožić, M. (2016). Op. cit., S. 44.

/39/ Bodrožić, M. (2016). Op. cit., S. 45.

/40/ Bodrožić, M. (2016). Op. cit., S. 41.

/41/ Bodrožić, M. (2016). Op. cit., S. 41.

/42/ Bodrožić, M. (2016). Op. cit., S. 80.

/43/ Bodrožić, M. (2016). Op. cit., S. 81.

/44/ Baar, Anna (42018). Die Farbe des Granatapfels.

Göttingen: Wallstein Verlag, S. 32.

/45/ Baar, Anna ( $\left.{ }^{4} 2018\right)$. Op. cit., S. 43.

/46/ Baar, Anna (42018). Op. cit., S. 60.

/47/ Baar, Anna ( $\left.{ }^{4} 2018\right)$. Op. cit., S. 43.

/48/ Baar, Anna ( $\left.{ }^{4} 2018\right)$. Op. cit., S. 88.

/49/ Baar, Anna (42018). Op. cit., S. 121.

/50/ Baar, Anna ("2018). Op. cit., S. 193-194.

/51/ Baar, Anna (42018). Op. cit., S. 220.

/52/ Baar, Anna ( $\left.{ }^{4} 2018\right)$. Op. cit., S. 221.

/53/ Pilic, D. (2015). Sommer vorm Balkan. Mein Leben zwischen Alpen und Adria. München: Wilhelm Goldmann Verlag, eBook, S. 8.

/54/ Pilic, D. (2015). Op. cit., S. 9.

/55/ Pilic, D. (2015). Op. cit., S. 9.

/56/ Pilic, D. (2015). Op. cit., S. 11.

/57/ Pilic, D. (2015). Op. cit., S. 14.

/58/ Pilic, D. (2015). Op. cit., S. 164.

/59/ Pilic, D. (2015). Op. cit., S. 195.

/60/ Pilic, D. (2015). Op. cit., S. 195.

/61/ Pilic, D. (2015). Op. cit., S. 24.

/62/ Baar, Anna ( $\left.{ }^{4} 2018\right)$. Op. cit., S. 44.

/63/ Baar, Anna ( $\left.{ }^{4} 2018\right)$. Op. cit., S. 41.

/64/ Baar, Anna ( $\left.{ }^{4} 2018\right)$. Op. cit., S. 45.

/65/ Baar, Anna ( $\left.{ }^{4} 2018\right)$. Op. cit., S. 73.

/66/ Baar, Anna (42018). Op. cit., S. 159.

/67/ Baar, Anna (42018). Op. cit., S. 202-203.

/68/ Baar, Anna (42018). Op. cit., S. 229.

/69/ Baar, Anna ( $\left.{ }^{4} 2018\right)$. Op. cit., S. 106.

/70/ Pilic, D. (2015). Op. cit., S. 82.

/71/ Pilic, D. (2015). Op. cit., S. 70.
/72/ Pilic, D. (2015). Op. cit., S. 46.

/73/ Pilic, D. (2015). Op. cit., S. 108.

/74/ Pilic, D. (2015). Op. cit., S. 109.

/75/ Bodrožić, M. (2016). Op. cit., S. 9.

/76/ Baar, Anna (42018). Op. cit., S. 14.

/77/ Baar, Anna (42018). Op. cit., S. 15.

/78/ Baar, Anna ( $\left.{ }^{4} 2018\right)$. Op. cit., S. 245.

/79/ Baar, Anna ( $\left.{ }^{4} 2018\right)$. Op. cit., S. 17.

/80/ Baar, Anna (2018). Op. cit., S. 17.

Literatur

Primärliteratur

1. Baar, Anna (42018). Die Farbe des Granatapfels. Göttingen: Wallstein Verlag.

2. Bodrožić, M. (2016). Sterne erben, Sterne färben. Meine Ankunft in Wörtern. München: btb Verlag, eBook.

3. Pilic, D. (2015). Sommer vorm Balkan. Mein Leben zwischen Alpen und Adria. München: Wilhelm Goldmann Verlag, eBook.

Sekundärliteratur

1. Abels, H. (32017). Identität. Über die Entstehung des Gedankens, dass der Mensch ein Individuum ist, den nicht leicht zu verwirklichenden Anspruch auf Individualität und Kompetenzen, Identität in einer riskanten Moderne zu finden und zu wahren. 3., aktualisierte und erweiterte Auflage. Wiesbaden: Springer VS.

2. Bhabha, H. K. (2011). Die Verortung der Kultur. Mit einem Vorwort von E. Bronfen. Übers. von M. Schiffmann und J. Freundl. Tübingen: Stauffenburg Verlag.

3. Bhabha, H. K. (2012). Über kulturelle Hybridität. Tradition und Übersetzung. Aus dem Englischen von Kathrina Menke. Wien-Berlin: Verlag Turia+Kant.

4. Eickelpasch, R., Rademacher, C. (2004). Identität. Bielefeld: transcript Verlag.

5. Frey, H. P., Haußer, K. (1987). Entwicklungslinien sozialwissenschaftlicher Identitätsforschung. In: Frey, H. P., Haußer K. (Hg.): Identität. Entwicklungen psychologischer und soziologischer Forschung. Stuttgart: Enke. (Der Mensch als soziales und personales Wesen. Bd. 7), S. 3-26.

6. Hofmann, M. (2006). Interkulturelle Literaturwissenschaft. Eine Einführung. Paderborn: Wilhelm Fink Verlag.

7. Keupp, H. (o. J.). Identität. Verfügbar unter: https://www.spektrum.de/lexikon/psychologie/identitaet/6968 [Zugriff am: 15. 4. 2020].

8. Lohauß, P. (1995). Moderne Identität und Gesellschaft. Theorien und Konzepte. Opladen: Leske + Budrich.

9. Metzeltin, M., Wallmann, T. (2010). Wege zur Europäischen Identität. Individuelle, nationalstaatliche und supranationale Identitätskonstrukte. Berlin: 
Frank \& Timme Verlag für wissenschaftliche Literatur.

10. Nünning, A. (Hrsg.). (2005). Grundbegriffe der Kulturtheorie und Kulturwissenschaften. StuttgartWeimar: Verlag J. B. Metzler.

11. Rădulescu, Raluca (2012). Hybride Identitäten zwischen Wortlandschaften. Marica Bodrožićs Prosaband „Sterne erben, Sterne färben“. In: Ger- manica, 51, S. 63-74. Verfügbar unter: https://journals.openedition.org/germanica/1986 [Zugriff am: 19. 4. 2020].

12. Wolting, M. (2017). „Identität kann nur als ein Problem existieren " - Zu den Identitätskonstruktionen in der Gegenwartsliteratur. Einleitung“. In: Wolting, M. (Hg.): Identitätskonstuktionen in der deutschen Gegenwartsliteratur. Göttingen: V\&R unipress, S. 9-18. 